\title{
Avaliação morfológica de Ananas comosus var. erectifolius (L. B. Smith) Coppens \&
}

\section{F. Leal-Bromeliacea}

\author{
Morphologica evaluation of Ananas comosus var. erectifolius (L. B. Smith) Coppens \& F. Leal- \\ Bromeliacea \\ Evaluación morfológica de Ananas comosus var. erectifolius (L. B. Smith) Coppens y F. Leal- \\ Bromeliacea
}

Recebido: 20/04/2021 | Revisado: 28/04/2021 | Aceito: 13/07/2021 | Publicado: 24/07/2021

Meiciane Ferreira Campelo
ORCID: https://orcid.org/0000-0001-7511-4377
Universidade Federal do Pará, Brasil
E-mail: meicianecampelo@ gmail.com
Osmar Alves Lameira
ORCID: https://orcid.org/0000-0001-8370-8562
Empresa Brasileira de Pesquisa Agropecuária Amazônia Oriental, Brasil
E-mail: osmar.lameira @embrapa.br
Ruanny Karen Vidal Pantoja Portal Moreira
ORCID: https://orcid.org/0000-0002-9566-3741
Universidade Federal do Pará, Brasil
E-mail: ruanny_vidal@ hotmail.com
Allan Christiam Santos Ramires
ORCID: https://orcid.org/0000-0002-0953-7221
Universidade Federal Rural da Amazônia, Brasil
E-mail: allanramires15@gmail.com

\section{Resumo}

O objetivo desse trabalho foi determinar descritores morfológicos de acessos de curauá através de caracteres morfológicos visando à avaliação dos germoplasmas, na Embrapa Amazônia Oriental, em Belém, PA. Foram estudados sete caracteres morfológicos quantitativos de 42 acessos de curauá, totalizando 210 indivíduos, conservados in vivo no Horto de Plantas Medicinais. Os dados foram submetidos as analises multivariadas, realizado a seleção direta e seleção com reanálise agrupadas em dois métodos e com base na distância Euclidiana média utilizada na formação dos agrupamentos pelos métodos Unweighted Paired Group Method Using ArithmeticAverages -UPGMA de otimização de Tocher e para análise de componentes principais. Dos caracteres avaliados nenhum se mostrou redundante, logo não houve descartes. Com base na distância Euclidiana média apresentaram três menores distâncias 0,052; 0,084 e 0,085 entre pares de acessos e permitiram separá-los em três grupos divergentes pelo método UPGMA e pelo método de Tocher houve a formação de quatro grupos divergentes. Os acessos de Ananas comosus var.erectifolius possuem variabilidade morfológica de acordo com os sete caracteres quantitativos em estudo, sendo os caracteres comprimento da folha, altura da planta e número de folhas os que mais contribuíram para a divergência entre os acessos.

Palavras-chave: Curauá; Banco ativo de germoplasma; Caracterização; Variação morfológica.

\begin{abstract}
The objective of this work was to determine morphological descriptors of accessions of Curauá through morphological characters aiming at the evaluation of germplasms, at Embrapa Amazônia Oriental, in Belém, PA. Seven quantitative morphological characters of 42 accessions of Curauá were studied, totaling 210 individuals, conserved in I live in the Medicinal Plants Garden. The data were submitted to multivariate analyzes, direct selection and selection with reanalysis were carried out grouped in two methods and based on the average Euclidean distance used in the formation of the clusters by the methods Unweighted Paired Group Method Using ArithmeticAverages UPGMA for Tocher's optimization and for analysis of main components. Of the characters evaluated, none were shown to be reduntant, so there were no discards. Based on the average Euclidean distance, they presented three shortest distances $0.052 ; 0.084$ and 0.085 between pairs of accessions and allowed to separate them into three divergent groups by the UPGMA method and by the Tocher method there was the formation of four divergent groups. The accessions of Ananas comosus var.erectifolius have morphological variability according to the seven quantitative characters under study, with the characters leaf length, plant height and number of leaves being the main contributors to the divergence between the accessions.
\end{abstract}

Keywords: Curauá; Active germplasm bank; Description; Morphological variation. 


\begin{abstract}
Resumen
El objetivo de este trabajo fue determinar descriptores morfológicos de accesiones de curauá a través de caracteres morfológicos con el objetivo de la evaluación de germoplasmas, en Embrapa Amazônia Oriental, en Belém, PA. Se estudiaron siete caracteres morfológicos cuantitativos de 42 accesiones de curauá, totalizando 210 individuos, conservado en vivo en el Jardín de Plantas Medicinales. Los datos se sometieron a análisis multivariados, la selección directa y la selección con reanálisis se llevaron a cabo agrupados en dos métodos y con base en la distancia euclidiana promedio utilizado en la formación de los conglomerados por los métodos Método de grupo emparejado no ponderado mediante promedios aritméticos -UPGMA para optimización de Tocher para análisis de componentes principales. De los personajes evaluados, ninguno resultó ser redundante, por lo que no hubo descartes. Con base en la distancia euclidiana promedio, presentaron tres distancias más cortas $0.052 ; 0.084$ y 0.085 entre pares de accesiones y se permitió separarlas en tres grupos divergentes por el método UPGMA y por el método de Tocher se formaron cuatro grupos divergentes. Las accesiones de Ananas comosus var.erectifolius presentan variabilidad morfológica según los siete caracteres cuantitativos en estudio, siendo los caracteres longitud de hoja, altura de planta y número de hojas los principales contribuyentes a la divergencia entre las accesiones.
\end{abstract}

Palabras clave: Curauá; Banco de germoplasma activo; Descripción; Variación morfológica.

\title{
1. Introdução
}

Entre as espécies da região Amazônica com potencial para produção de fibras, destaca-se o curauá (Ananas comosus var.erectifolius (L.B. Smith) Coppens \& F. Leal). No Brasil e no exterior, a fibra de curauá é submetida a freqüentes pesquisas, que vêm apresentando resultados significativos, o que torna essa espécie a mais promissora entre as espécies produzidas na Amazônia brasileira (Cordeiro et al., 2016).Estudos recentes têm demonstrado o grande potencial desta planta como produtora de fibra de excelente qualidade, sendo utilizada na indústria automobilística e têxtil devido sua resistência, maciez e peso reduzido, podendo ainda ser utilizada como celulose e ração animal. Há uma crescente demanda de fibras do curauá por grupos empresariais preocupados principalmente na utilização de produtos naturais biodegradáveis, o que torna essa espécie estratégica para o estado e cria uma perspectiva de melhoria da qualidade de vida dos pequenos produtores (Lameira et al., 2003).

Em virtude disso, a Instituição Embrapa Amazônia Oriental localizada no município de Belém-PA, no ano de 2001 instalou o Banco Ativo de Germoplasma (BAG) de curauá com o objetivo de introduzir, coletar, conservar, caracterizar, documentar e disponibilizar o germoplasma dessa espécie (Pádua et. al., 2020) nesse banco os acessos coletados de diferentes procedências nos permite conduzir estudos na área de caracterização e avaliação morfológica.

Portanto, o uso de um Banco Ativo de Germoplasma tem como principais objetivos diminuir a erosão genética quando a pressão por uma determinada espécie está alta, conservar genes que possam ser de interesse futuro e possibilitar a utilização dos indivíduos ali depositados em programas de melhoramento (Rios, 2020). A avaliação do germoplasma é uma etapa importante, pois viabiliza seu uso e justifica a conservação da espécie, possibilitando avanços em estudos de variabilidade genética, assim como facilita a identificação de indivíduos superiores em relação a alguma característica específica, além de descrever semelhanças e diferenças entre acessos (Mata, 2010).

De acordo com Silva (2014), o estudo da diversidade genética disponibiliza informações fundamentais, em relação à caracterização, conservação e utilização dos recursos genéticos disponíveis. Outro aspecto é que a avaliação vegetal também é uma etapa fundamental no processo de criação de novos genótipos pelo melhoramento genético vegetal. Em que os descritores bem definidos, podem garantir características hereditárias próprias da nova variedade e podem fornecer os elementos para manutenção da pureza vegetal.

Em vista do que foi exposto, o objetivo desse trabalho foi determinar descritores morfológicos de acessos de curauá através de caracteres morfológicos visando à avaliação dos germoplasmas. 


\section{Metodologia}

Este trabalho aborda uma pesquisa de campo, sendo de método quantitativo de acordo com Pereira et al. (2018). Sendo realizado no BAG de Ananas comosus var. erectifolius (L. B. Smith) Coppens \& F. Leal, situado no Horto de Plantas Medicinais da Embrapa Amazônia Oriental, com altitude de $10 \mathrm{~m}$ e temperatura média anual de $30{ }^{\circ} \mathrm{C}$. O clima em Belém, segundo análise estatísticas da série histórica da cidade, apresenta precipitação anual média de 3.070 mm, entretanto, as chuvas se distribuem de maneira diferente durante o ano, formando um período muito chuvoso (dezembro a maio) e um período menos chuvoso (junho a novembro) Lira et al., (2020).

Foram avaliados 42 acessos de Ananas comosus (Tabela 1), todos conservados in vivo, cultivados em pleno sol e os tratos culturais e fitossanitários foram realizados de acordo com a necessidade da espécie. A identificação botânica foi realizada no Laboratório de Botânica da Embrapa Amazônia Oriental, e as exsicatas, encontram-se no Herbário IAN da mesma instituiçãocom os seguintes números: IAN 185100 - Ananas comosus var.erectifolius (cuaruá-roxo) e IAN 185101 Ananas comosus var. erectifolius (curauá-branco).Os acessos possuem procedência em seis municípios do Estado do Pará- Brasil, sendo sete de Alenquer, dois de Belém, um de Bragança, nove de Monte Alegre, dois de Ponta de Pedras e vinte e um de Santarém. (Alelo, 2021). Foram selecionadas cinco plantas por acesso de forma aleatória e marcados com fita.

Tabela 1. Procedência e nomenclatura de 42 acessos registrados no BAG de Ananas comosus var.erectifolius da Embrapa Amazônia Oriental.

\begin{tabular}{|c|c|c|}
\hline $\begin{array}{l}\text { Procedência } \\
\text { (Municípios) }\end{array}$ & Nomenclatura (ALELO) & Código Local \\
\hline Alenquer & $\begin{array}{l}\text { NLR 2200, AMR 2300, } \\
\text { HBR 2400, SRR 2500, } \\
\text { FMR 2600, AMB } 2301 \\
\text { HBB 2401 }\end{array}$ & $\begin{array}{l}\text { NLR ROXO, AMR ROXO } \\
\text { HBR ROXO, SRR ROXO, } \\
\text { FMR ROXO, AMB BRANCO } \\
\text { HBB BRANCO }\end{array}$ \\
\hline Belém & $\begin{array}{l}\text { EMBRAPA R 01, } \\
\text { EMBRAPA B } 01\end{array}$ & $\begin{array}{l}\text { EMBRAPA ROXO, } \\
\text { EMBRAPA BRANCO }\end{array}$ \\
\hline Bragança & BB 701 & BB BRANCO \\
\hline Monte Alegre & $\begin{array}{l}\text { FNR 2100, NMB 1301, } \\
\text { JPB 1401, EFB 1501, } \\
\text { JRB 1601, SAB 1701, } \\
\text { LAB 1801, NFB 1901, } \\
\text { ASB } 2001\end{array}$ & $\begin{array}{l}\text { FNR ROXO, NMB BRANCO, } \\
\text { JPB BRANCO, EFB BRANCO, } \\
\text { JRB BRANCO, SAB BRANCO, } \\
\text { LAB BRANCO, NFB BRANCO, } \\
\text { ASB BRANCO }\end{array}$ \\
\hline Ponta de Pedras & PPR 400, PPB 401 & PPR ROXO, PPB BRANCO \\
\hline Santarém & $\begin{array}{l}\text { MR 001, CR 200, MFR 800, } \\
\text { DR 500, BFR 600, MB 002, PEMATEC } \\
\text { R01, AB 101, } \\
\text { CB 201, TB 301, DB 501, } \\
\text { BFB 601, PAR 900, TR } 300 \\
\text { PEMATEC B02, RSR } 1100, \\
\text { ANR 1200, MFB 801, } \\
\text { PAB 901, RSB 1101, ANB } 1201\end{array}$ & $\begin{array}{l}\text { MR ROXO, CR ROXO, } \\
\text { MFR ROXO, DR ROXO, } \\
\text { BFR ROXO, MB BRANCO, } \\
\text { PEMATEC ROXO, } \\
\text { AB BRANCO, CB BRANCO, } \\
\text { TB BRANCO, DB BRANCO, BFB } \\
\text { BRANCO, PAR ROXO, } \\
\text { TR ROXO, PEMATEC BRANCO, } \\
\text { RSR ROXO, ANR ROXO, } \\
\text { MFB BRANCO, PAB BRANCO, RSB } \\
\text { BRANCO, ANB BRANCO }\end{array}$ \\
\hline
\end{tabular}


Para seleção de descritores e divergência genética entre os acessos os caracteres morfológicos quantitativos avaliados foram sete: altura da planta; largura da folha; comprimento da folha; número de perfilhos na base, número de perfilhos na base do fruto, número de perfilho no ápice e número de folhas. As medições foram realizadas com auxílio de régua e paquímetro.

Após tabulação dos dados, foi realizada análise segundo dois métodos com o auxílio do software estatístico R (2020) para identificação de possíveis caracteres quantitativos redundantes ou invariáveis para posterior seleção dos descritores, o primeiro método foi o de seleção direta, proposto por Jolliffe (1937) neste caso foram eliminados todos os caracteres que apresentarem maior coeficiente de ponderação em valor absoluto (autovetor) no componente principal de menor autovalor, partindo do último componente até aquele cujo autovalor não excedesse a 0,70. O segundo método foi a seleção com reanálise, sugerida por Cury (1993) em que para cada caráter sugerido para descarte, foi realizada nova análise com os caracteres remanescentes, examinando os coeficientes de correlação do caráter sugerido para descarte com os demais caracteres, sendo finalizada quando o caráter a ser descartado se mostrou altamente correlacionado com pelo menos um caráter já descartado. O descarte final foi realizado com base na informação obtida simultaneamente nos dois procedimentos.

Os dados dos caracteres selecionados foram empregados para a obtenção das dissimilaridades entre cada par de acessos com base na distância euclidiana média padronizada, no mesmo software, uma vez que os acessos encontram-se estabelecidos sem obedecer a nenhum delineamento experimental, assim como os caracteres em diferentes medidas e escalas, esta análise foi executada com base na média de cada caráter. A matriz de distância (dissimilaridade) obtida foi utilizada na formação da análise de componentes principais e dos agrupamentos pelos métodos UPGMA e de otimização de Tocher.

\section{Resultados e Discussão}

Dos sete caracteres avaliados segundo os dois procedimentos utilizados nenhum se mostrou redundante, logo não houve descartes. A eliminação de caracteres redundantes é uma decisão vantajosa, pois reduz o trabalho sem ocasionar perda na precisão da caracterização, especialmente se esses caracteres forem de difícil mensuração e apresentarem baixa variabilidade (Daher, 1993). Ressalta-se, porém, que após o descarte, o conjunto de caracteres reduzido deve se mostrar efetivo na representação da variação total (Torres Filho, 2008). Contudo, neste trabalho foi realizado o procedimento de seleção direta, proposto por Jolliffe (1937) a seleção com reanálise, sugerida por Cury (1993), entretanto os testes não identificaram redundância que justifiquem descartes.

A matriz de dissimilaridade obtida entre os pares de 42 acessos de Ananas comosus gerada com base em sete caracteres quantitativos revela maior semelhança, ou seja, maior similaridade com a menor distância $(0,052)$ entre os acessos NFB BRANCO $\times$ JRB BRANCO ambos provenientes de Monte Alegre, a segunda menor distância $(0,084)$ entre os pares MFB BRANCO x AB BRANCO ambos provenientes de Santarém e a terceira menor distância $(0,085)$ entre os pares NMB BRANCO x JPB BRANCO ambos provenientes de Monte Alegre. A diversidade genética pode estar associada à distância geográfica, neste estudo, os pares de acessos que apresentaram a menor dissimilaridade, ou seja, os mais semelhantes entre si possuem mesma procedência. Fatores, como a seleção, deriva genética, fluxo gênico, entre outros, podem atuar de forma conjunta ou isolada, em diferentes intensidades e determinar a existência ou não de algum paralelismo entre essas medidas (Alves, 2002).

Com base no método UPGMA, os 42 acessos formaram três grupos divergentes: grupo I contendo apenas o acesso SRRROXO, o II composto pelos acessos MR, CR, TR, DR, BFR, MFR, PAR, RSR, ANR, FNR, HBR, AR, NLR, AMR , FMR, PPR, EMBRAPA R , BB e o grupo III contendo os demais acesso MB, AB, CB , TB, DB, BFB , PEMATEC B, MFB, PAB , RSB , ANB , NMB , JPB , AMB, HBB , EFB , JRB, SAB, PPB, EMBRAPA B, LAB, NFB, ASB (Figura 1).

Pode-se perceber no agrupamento destes acessos, que o grupo II abrange entre os 18 acessos que o compõe 17 acessos pertencente ao tipo roxo, enquanto que o grupo III e composto em sua totalidade por 23 acessos do tipo Branco, as 
denominações Branco e Roxo se refere à coloração das folhas da planta do curauá, logo nota-se a importância da avaliação em conjunto de caracteres quantitativos e qualitativos.A análise conjunta dos dados quantitativos e qualitativos permite a unificação de todas as informações, proporcionando maior eficiência na determinação da divergência genética entre os acessos de uma coleção e constituindo-se em alternativa viável e ferramenta importante para o conhecimento do germoplasma (Maria da Cruz et al., 2010). Um dos principais objetivos dos programas de melhoramento genético é a seleção de plantas com características superiores para que isto ocorra, é fundamental o conhecimento das correlações entre os caracteres qualitativos, uma vez que estes podem impactar positiva ou negativamente o progresso genético (Torres, 2016).

Figura 1. Dendograma gerado pelo método UPGMA a partir das dissimilaridades obtidas entre 42 acessos de Ananas comosus var.erectifolius conservados no BAG da Embrapa Amazônia Oriental, com base em sete caracteres morfológicos.

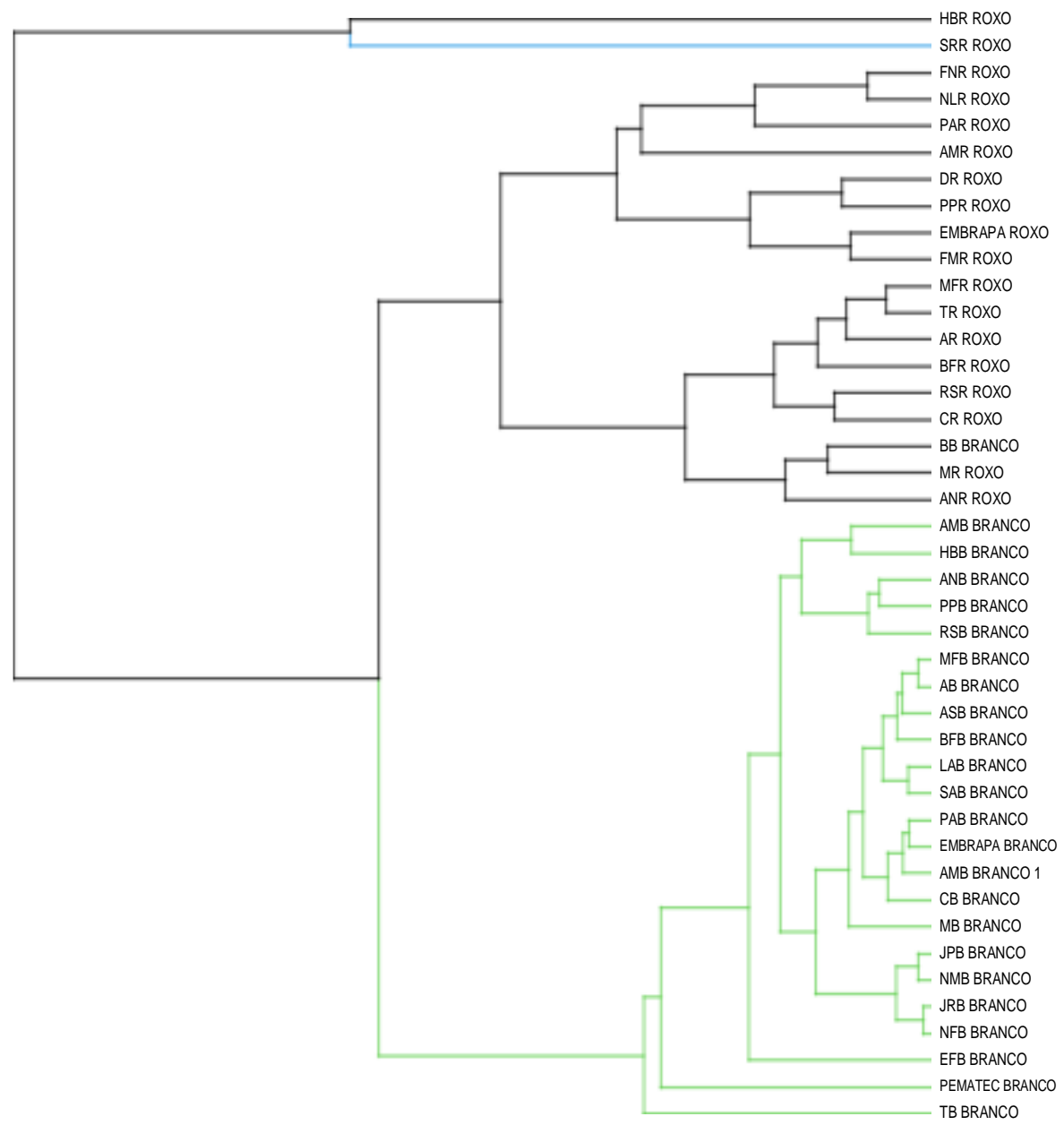

Fonte: Autores.

Pelo método de Tocher houve a formação de quatro grupos de genótipos divergentes entre os 42 acessos. No grupo I esta presente apenas o acesso HPR ROXO, grupo II composto por SRR ROXO, PPR ROXO e DR ROXO, grupo III formado por TR ROXO, MFR ROXO, AR ROXO, BFR ROXO, RSR ROXO. CR ROXO, ANR ROXO, FNR ROXO, NLR ROXO, PAR ROXO, FMR ROXO, EMBRAPA ROXO e grupo IV agrupando os demais 26 acessos (Tabela 2). No método de otimização de Tocher, realiza-se a partição do conjunto de acessos em subgrupos, adotando o critério de que a média das medidas de dissimilaridade, dentro de cada grupo, deve ser menor que as distâncias médias entre quaisquer grupos 
(Vasconcelos et al., 2012).Observou-se alguma semelhança na formação dos grupos com a análise pelo método do UPGMA, ressalta-se a formação dos grupos I,II, e III com exclusivamente acessos com denominação roxo e o grupo IV com quase sua totalidade com acessos de denominação branca exceto pelos acessos MR ROXO e AMR ROXO.

Tabela 2. Grupos formados pelo método de Tocher entre os 42 acessos de Ananas comosus var. erectifolius com base em sete caracteres morfológicos-Distância Euclidiana média padronizada.

\begin{tabular}{|c|c|c|c|c|c|c|c|}
\hline Grupos & & & & Acessos & & & \\
\hline $\mathbf{I}$ & $\begin{array}{l}\text { HBR } \\
\text { ROXO }\end{array}$ & & & & & & \\
\hline II & $\begin{array}{l}\text { PPR } \\
\text { ROXO }\end{array}$ & $\begin{array}{l}\text { DR } \\
\text { ROXO }\end{array}$ & $\begin{array}{l}\text { SRR } \\
\text { ROXO }\end{array}$ & & & & \\
\hline III & $\begin{array}{l}\text { TR } \\
\text { ROXO }\end{array}$ & $\begin{array}{l}\text { MFR } \\
\text { ROXO }\end{array}$ & $\begin{array}{l}\text { AR } \\
\text { ROXO }\end{array}$ & $\begin{array}{l}\text { BFR } \\
\text { ROXO }\end{array}$ & $\begin{array}{l}\text { RSR } \\
\text { ROXO }\end{array}$ & $\begin{array}{l}\text { CR } \\
\text { ROXO }\end{array}$ & $\begin{array}{l}\text { ANR } \\
\text { ROXO }\end{array}$ \\
\hline & $\begin{array}{l}\text { FNR } \\
\text { ROXO }\end{array}$ & $\begin{array}{l}\text { NLR } \\
\text { ROXO }\end{array}$ & $\begin{array}{l}\text { PAR } \\
\text { ROXO }\end{array}$ & $\begin{array}{l}\text { FMR } \\
\text { ROXO }\end{array}$ & $\begin{array}{l}\text { EMBRAPA } \\
\text { ROXO }\end{array}$ & & \\
\hline IV & $\begin{array}{l}\text { NFB } \\
\text { BRANCO }\end{array}$ & $\begin{array}{l}\text { JRB } \\
\text { BRANCO }\end{array}$ & $\begin{array}{l}\text { JPB } \\
\text { BRANCO }\end{array}$ & $\begin{array}{l}\text { NMB } \\
\text { BRANCO }\end{array}$ & $\begin{array}{l}\text { SAB } \\
\text { BRANCO }\end{array}$ & $\begin{array}{l}\text { MFB } \\
\text { BRANCO }\end{array}$ & $\begin{array}{l}\mathrm{AB} \\
\mathrm{BRANCO}\end{array}$ \\
\hline & $\begin{array}{l}\text { LAB } \\
\text { BRANCO }\end{array}$ & $\begin{array}{l}\text { ASB } \\
\text { BRANCO }\end{array}$ & $\begin{array}{l}\text { BFB } \\
\text { BRANCO }\end{array}$ & $\begin{array}{l}\text { PAB } \\
\text { BRANCO }\end{array}$ & $\begin{array}{l}\text { AMB } \\
\text { BRANCO }\end{array}$ & $\begin{array}{l}\text { EMBRAPA } \\
\text { BRANCO }\end{array}$ & $\begin{array}{l}\text { MB } \\
\text { BRANCO }\end{array}$ \\
\hline & $\begin{array}{l}\text { CB } \\
\text { BRANCO }\end{array}$ & $\begin{array}{l}\text { HBB } \\
\text { BRANCO }\end{array}$ & $\begin{array}{l}\text { PPB } \\
\text { BRANCO }\end{array}$ & $\begin{array}{l}\text { RSB } \\
\text { BRANCO }\end{array}$ & $\begin{array}{l}\text { ANB } \\
\text { BRANCO }\end{array}$ & $\begin{array}{l}\text { AMB } \\
\text { BRANCO }\end{array}$ & $\begin{array}{l}\text { EFB } \\
\text { BRANCO }\end{array}$ \\
\hline & $\begin{array}{l}\text { BB } \\
\text { BRANCO }\end{array}$ & $\begin{array}{l}\text { PEMATEC } \\
\text { BRANCO }\end{array}$ & $\begin{array}{l}\text { TB } \\
\text { BRANCO }\end{array}$ & $\begin{array}{l}\text { MR } \\
\text { ROXO }\end{array}$ & $\begin{array}{l}\text { AMR } \\
\text { ROXO }\end{array}$ & & \\
\hline
\end{tabular}

Fonte: Autores.

O comprimento da folha, altura da planta e número de folhas, foram os caracteres que mais contribuíram para a divergência entre os 42 acessos com percentuais de contribuição igual a 18,69\%; 18,64 e 15,13\% respectivamente (Tabela 3). Resultados semelhantes foram observados por Lameira et al., (2020) realizando avaliação de 10 acessos de Ananas comosus de diversas procedências, afirma que o comprimento da folha apresenta maior contribuição para divergência entre os germoplasmas. Assis e Giulieti (1999) em um estudo comparativo de populações de Rutaceae, afirmaram que as variações associadas ao comprimento e largura das folhas, estão expressas na variação das folhas, na qual variam morfologicamente.

O componente que menos contribuiu foi o caráter largura da folha 6,08\%. A análise de componentes principais vem se destacando como a metodologia mais empregada em bancos e ou coleções de germoplasma, pois, identificar os caracteres mais importantes na contribuição de variação total disponível entre os indivíduos analisados (Pereira, 1989), e fornece indicação para eliminar os que pouco contribuem, desta forma proporcionando redução na mão de obra, no tempo e custos das atividades da avaliação e caracterização do banco ativo de germoplasma do curauá (Lameira et al., 2020). 
Tabela 3. Contribuição relativa de sete caracteres para a dissimilaridade de 42 acessos de Ananas comosus conservados no BAG da Embrapa Amazônia Oriental, com base em sete caracteres morfológicos.

\begin{tabular}{ll}
\hline Caráter & Contribuição (\%) \\
\hline Altura planta & 18,64 \\
Largura folha & 6,08 \\
Comprimento folha & 18,69 \\
Perfilho base & 12,82 \\
Perfilho ápice & 14,16 \\
$\mathrm{~N}^{\circ}$ perfilho base fruto & 14,45 \\
$\mathrm{~N}^{\circ}$ de folhas & 15,13 \\
\hline
\end{tabular}

Fonte: Autores.

Embora a caracterização morfoagronômica seja vista como uma medida indireta da variabilidade total dos acessos, Moreira et al., (2014) foi capaz de detectar acentuada diversidade entre todos os caracteres e entre todos os acessos de curauá. Assim como o presente estudo de caracterização morfológica identificou através dos sete caracteres avaliados variabilidade entre os germoplasmas de curauá. Lameira et al., (2020) em estudo de caracteres quantitativos de Ananas comosus sugere complemento com estudos posteriores de caracteres qualitativos.

\section{Conclusão}

Este estudo avaliou a variabilidade dos caracteres quantitativos altura da planta, largura da folha, comprimento da folha, número de perfilhos na base, número de perfilhos na base do fruto, número de perfilho no ápice, número de folhas, todos apresentaram contribuição para diferenciação entre acessos de Ananas comosus.

Identificou e agruparam os descritores, os que mais contribuíram para a divergência entre os acessos foram comprimento da folha, altura da planta e número de folhas, mostrando-se importantes na avaliação de germoplasma de curauá. Recomenda-se a soma de estudos de caracteres morfológicos qualitativos para a espécie.

Estas informações são cruciais para a espécie, auxilia no conhecimento e uso da variabilidade génetica e servirão de base para a seleção de indivíduos de interesse em programas de melhoramento, além de fornecer informações fundamentais que facilitará a identificação de espécimes em ecossistemas nativos ou plantados.

\section{Agradecimentos}

Os autores agradecem à Coordenação de Aperfeiçoamento de Pessoal de Nível Superior (CAPES) pelo apoio e bolsa de estudo de Doutorado, à Embrapa Amazônia Oriental por ceder o local e a estrutura, e ao Programa de Doutorado da Rede Bionorte- Biodiversidade e Biotecnologia.

\section{Referências}

Alves, R. M. (2002). Caracterização genética de populações de cupuaçuzeiro, Theobroma grandiflorum (Willd. ex. Spreng.) Schum., por marcadores microssatélites e descritores botânico-agronômicos (Doctoral dissertation, Universidade de São Paulo).

Assis, M. C. D., \& Giulietti, A. (1999). Diferenciação morfológica e anatômica em populações de" ipecacuanha"-Psychotria ipecacuanha (Brot.) Stokes (Rubiaceae). Brazilian Journal of Botany, 22(2), 205-216.

Cordeiro, I. M. C. C., Oliveira Junior, M. C. M. D., Gazel-Filho, A. B., Barros, P., Lameira, O. A., \& Oliveira, F. D. A. (2016). Crecimiento del Schizolobium parahyba var. amazonicum cultivado en presencia de Ananas comosus var. erectifolius en Pará, Brasil. Agrociencia, 50(1), 79-88. 
Cury, R. (1993). Dinâmica evolutiva e caracterizaçao de germoplasma de mandioca (Manihot esculenta, Crantz) na agricultura autóctone do Sul do Estado de Sao Paulo (Doctoral dissertation, Universidade de São Paulo).

Daher, R. (1993). Diversidade morfológica e isoenzimática em capim elefante (Pennisetum purpureum Schum.).110 $\mathrm{f}$ (Doctoral dissertation, Dissertação (Mestrado em Genética e Melhoramento de Plantas)-Universidade Federal de Viçosa, Viçosa, MG).

Embrapa. (2021). Embrapa Recursos Genéticos E Biotecnologia. Núcleo de Tecnologia da Informação (NTI). AleloVegetal.

Jolliffe, I. T. (1973). Discarding variables in a principal componentanalysis. II: Real data. Journal of the Royal Statistical Society: Series C (Applied Statistics), 22(1), 21-31.

Lameira, O. A., Cordeiro, I. M. C. C., \& Pires, H. C. G. (2020). Avaliação dos descritores morfoagronômico e morfoanatomia da lâmina foliar de Pilocarpus microphyllus Stapf Ex Wardleworth,Rutaceae, Ananas comosus var.erectifolius(lb smith)coppens\&f.leal,Bromeliacea e Psychotria ipecacuanha(brot.) stokes, Rubiaceae. Editora APPRIS.

Lameira, O. A., Reis, I. N. R., \& Cordeiro, I. M. C. C. (2003). Otimização da propagação "in vitro" de curauá (Ananas erectifolius L.B.Smith). Revista Biotecnologia Ciência e Desenvolvimento, 78-81.

Lira, B. R. P., Lopes, L. D. N. A., das Chaves, J. R., Santana, L. R., \& Fernandes, L. L. (2020). Identificação de Homogeneidade, Tendência e Magnitude da Precipitação em Belém (Pará) entre 1968 e 2018. Anuário do Instituto de Geociências, 43(4), 426-439.

Maria da Cruz, C. L., Gonçalves, L. S., Sudré, C. P., Rodrigues, R., Antonio, T. D., Júnior, A., \& Pereira, T. N. (2010). Algoritmo de Gower na estimativa da divergência genética em germoplasma de pimenta. Hortic. bras, 28(2).

Mata, T. L. D. (2010). Diversidade genética em germoplasma de arroz filipino identificada por marcadores moleculares e caracteres agromorfológicos (Doctoral dissertation, Universidade de São Paulo).

Moreira, C. M., Bertolucci, S. K. V., Lameira, O. A., Rocha, T. T., Andrade, H. B., Gavilanes, M. L., \& Pinto, J. E. B. P. (2014). Caracterização morfológica de acessos de curauá do banco ativo de germoplasma da Embrapa Amazônia Oriental. Revista Agrogeoambiental, 7(2).

Pádua, J. G., Albuquerque, M., \& de Mello, S. C. M. (2020). Bancos e coleções de germoplasma da Embrapa: Conservação e uso. Embrapa Recursos Genéticos e Biotecnologia-Documentos (INFOTECA-E).

Pereira, A. S., Shitsuka, D. M., Parreira, F. J. \& Shitsuka, R. (2018). Metodologia da pesquisa científica. UFSM. 119p. https://repositorio.ufsm.br/bitstream /handle/1 /1582 4/Lic_Computacao_Metodologia-Pesquisa-Cientifica.pdf?sequence=1.

Pereira V. A. Utilização de análise multivariada na caracterização de germoplasma de mandioca ( ManihotesculentaCrantz.) [tese]. Piracicaba: Escola Superior de Agricultura Luiz de Queiroz, Universidade de São Paulo.

R Core Team (2020). R: A languageandenvironment for statisticalcomputing. R Foundation for StatisticalComputing, Vienna, Austria. URL https://www.Rproject.org/.

Rios, R. D. M. (2020). Caracterização morfoagronômica e molecular de acessos de açaí coletados na Amazônia Oriental brasileira. Embrapa AmapáTese/dissertação (ALICE).

Silva, M. L. D. (2014). Cultivo agroflorestal de Psychotria Ipecacuanha (Brot.) Stokes no território do baixo sul da Bahia. Dissertação (Mestrado em Recursos Genéticos Vegetais) - Universidade Estadual de Feira de Santana, Feira de Santana.

Torres Filho, J. (2008). Morpho-agronomic characterization, selection of germplasm descriptors and association between genetic divergence and heterosis in melon plants. 150 F. TESE (DOUTORADO EM AGRICULTURA TROPICAL) - Universidade Federal Rural do Semi-Árido, Mossoró, 2008.

Torres, F. E., Valle, C. B. D., Lempp, B., Teodoro, P. E., Santos, A. D., \& Ribeiro, L. P. (2016). Contribuição dos caracteres de qualidade da forragem ao teor de proteína bruta em Urochloa brizantha. Pesquisa Agropecuária Brasileira, 51(3), 284-287.

Vasconcelos, C. S. D., Barbieri, R. L., Neitzke, R. S., Priori, D., Fischer, S. Z., \& Mistura, C. C. (2012). Determinação da dissimilaridade genética entre acessos de Capsicum chinense com base em características de flores. Revista Ceres, 59(4), 493-498. 